\title{
What are cohort studies?
}

\section{David Barrett, ${ }^{\oplus 1}$ Helen Noble ${ }^{2}$}

10.1136/ebnurs-2019-103183

${ }^{1}$ Faculty of Health Sciences, University of Hull, Hull, UK

${ }^{2}$ School of Nursing and

Midwifery, Queen's University

Belfast, Belfast, UK

Correspondence to:

Dr David Barrett, Faculty of

Health Sciences, University

of Hull, Hull HU6 7RX, UK; D.I.

Barrett@hull.ac.uk
In 1951, Richard Doll and Austin Bradford-Hill commenced a ground-breaking research project by writing to all registered doctors in the UK to ask about their smoking habits. The British Doctors Study recruited and followed-up over 40000 participants, monitoring mortality rates and causes of death over the subsequent years and decades. Even by the time of the first set of preliminary results in 1954, there was evidence to link smoking with lung cancer and increased mortality. ${ }^{1}$ Over the following decades, the study provided further definitive evidence of the health risks from smoking, and was extended to explore other causes of death (eg, heart disease) and other behavioural variables (eg, alcohol intake).

The Doctors Health Survey is one of the largest, most ambitious and best-known cohort studies and demonstrates the value of this approach in supporting our understanding of disease risk. However, as a method, cohort studies can have much wider applications. This article provides an overview of cohort studies, identifying the opportunities and challenges they present to researchers, and the role they play in developing the evidence base for nursing and healthcare more broadly.

\section{What are cohort studies?}

Cohort studies are a type of longitudinal study-an approach that follows research participants over a period of time (often many years). Specifically, cohort studies recruit and follow participants who share a common characteristic, such as a particular occupation or demographic similarity. During the period of follow-up, some of the cohort will be exposed to a specific risk factor or characteristic; by measuring outcomes over a period of time, it is then possible to explore the impact of this variable (eg, identifying the link between smoking and lung cancer in the British Doctors Study.) Cohort studies are, therefore, of particular value in epidemiology, helping to build an understanding of what factors increase or decrease the likelihood of developing disease.

The British Doctors Study is just one of many largescale, long-term cohort studies carried out to enhance understanding of the causes of disease and to help to develop evidence-based guidelines for healthier living. For example, the Framingham Heart Study-which commenced in 1948 and is now following up a third generation that includes grandchildren of the original cohort of participants from a Massachusetts town-has provided extensive data on the risk factors for cardiovascular disease and underpinned international guidelines on prevention. ${ }^{2}$ Similarly, the Nurses' Health Survey (https://www.nurseshealthstudy.org)-also now in its third generation-has recruited approximately 280000 participants across the USA. Through monitoring of risk factors and outcomes over 40 years, this study has provided some important insights into health risks, such as obesity increasing the risk of some cancers and shift work being linked with an increased likelihood of developing chronic diseases. ${ }^{3}$

Though the most high-profile types of cohort studies are usually related to large epidemiological research studies, they are not the only application of this method. Within nursing research, cohort studies have focused on the progress of nurses through their education and careers. Li et al-as part of the European NEXT study group-recruited almost 6500 female nurses who, at the time of recruitment, had no intention to leave the profession. The study followed the cohort up for a year, identifying that $8 \%$ developed the intention to leave nursing, often due to issues such as poor salary or limited promotion prospects. ${ }^{4}$

Usually, cohort studies should adopt a purely observational approach. However, some research is labelled as a cohort study while exploring the effectiveness of specific interventions. For example, Lansperger et al explored nurse practitioner (NP)-led critical care in a large university hospital in the USA. They collected data on all patients who were admitted to the intensive care unit over a 3-year period. Patients from this cohort were cared for by teams led by either doctors or NPs, and outcomes (primarily 90-day mortality) were monitored. By comparing the groups, the researchers established that outcomes were similar regardless of whether patient care was led by a doctor or an NP. ${ }^{5}$

\section{Strengths and weaknesses of cohort studies}

Cohort studies are an effective and robust method of establishing cause and effect. As they are usually large in size, researchers are able to draw confident conclusions regarding the link between risk factors and disease. In many cases, because participants are often free of disease at the commencement of the study, cohort studies are particularly useful at identifying the timelines over which certain behaviours can contribute to disease.

However, the nature of cohort studies can cause challenges. Collecting prospective data on thousands of participants over many years (and sometimes decades) is complex, time-consuming and expensive. Participants may drop out, increasing the risk of bias; equally, it is possible that the behaviour of participants may alter because they are aware that they are part of a study cohort. The analysis of data from these large-scale studies is also complex, with large numbers of confounding variables making it difficult to link cause and effect. Where cohort (or 'cohort-like') studies link to a specific intervention (as in the case of the Lansperger et al study into nursing practitioner-led critical care $^{5}$ ), the lack of randomisation to different arms of the study makes the approach less robust than randomised controlled trials.

One way of making a cohort study less time-consuming is to carry it out retrospectively. This is a more pragmatic approach, as it can be completed more quickly using historical data. For example, Wray et al used a retrospective cohort study to identify factors that 
were associated with non-continuation of students on nursing programmes. By exploring characteristics in five previous cohorts of students, they were able to identify that factors such as being older and/or local were linked to higher levels of continuation. ${ }^{6}$

However, this retrospective approach increases the risk of bias in the sampling of the cohort, with greater likelihood of missing data. Retrospective cohort studies are also weakened by the fact that the data fields available are not designed with the study in mind-instead, the researcher simply has to make use of whatever data are available, which may hinder the quality of the study.

\section{Reporting and critiquing of cohort studies}

When reporting a cohort study, it is recommended that STROBE guidance ${ }^{7}$ is followed. STROBE is an international, collaborative enterprise which includes experts with experience in the organisation and of dissemination of observational studies, including cohort studies. The aim is to STrengthen the Reporting of OBservational studies in Epidemiology. The STROBE checklist for cohort studies - available at https://www.strobe-statement.org/ fileadmin/Strobe/uploads/checklists/STROBE_checklist_ v4_combined.pdf- includes detail related to the introduction/methods/results/discussion of the study.

Critical appraisal of any cohort study is essential to identify the strengths and weaknesses of the study and to determine the usefulness and validity of the study findings. Components of critical appraisal in relation to cohort studies include evaluation of the study design in relation to the research question, assessment of the methodology, suitability of statistical methods used, conflicts of interest and how relevant the research is to practice. ${ }^{8-10}$

\section{Conclusion}

Cohort studies are the cornerstone of epidemiological research, providing an understanding of risk factors for disease based on findings in thousands of participants over many years. Disease prevention guidelines used by nurses and other healthcare professionals across the globe are based on the evidence from high-profile studies, such as the British Doctors Study, the Framingham Heart Study and the Nurses' Health Study. However, cohort studies offer opportunities outside epidemiology: in nursing research, the approach is useful in exploring areas such as factors that influence students' progression through their programme or nurses' progression through their career.

This approach to research does bring with it some important challenges-often related to their size, complexity and longevity. However, with careful planning and implementation, cohort studies can make valuable contributions to the development of evidence-based healthcare.

\section{Competing interests None declared.}

Patient consent for publication Not required.

Provenance and peer review Commissioned; internally peer reviewed.

๑ Author(s) (or their employer(s)) 2019. No commercial re-use. See rights and permissions. Published by BMJ.

\section{References}

1. Doll R, Hill AB. The mortality of doctors in relation to their smoking habits; a preliminary report. Br Med J 1954;1:1451-5.

2. Tsao CW, Vasan RS. Cohort profile: the Framingham heart study (FHS): overview of milestones in cardiovascular epidemiology. Int J Epidemiol 2015;44:1800-13.

3. Colditz GA, Philpott SE, Hankinson SE. The impact of the nurses' health study on population health: prevention, translation, and control. Am J Public Health 2016;106:1540-5.

4. Li J, Galatsch M, Siegrist J, et al. Reward frustration at work and intention to leave the nursing profession-prospective results from the European longitudinal NEXT study. Int J Nurs Stud 2011;48:628-35.

5. Landsperger JS, Semler MW, Wang L, et al. Outcomes of nurse practitioner-delivered critical care: a prospective cohort study. Chest 2016;149:1146-54.

6. Wray J, Aspland J, Barrett D, et al. Factors affecting the programme completion of PRE-REGISTRATION nursing students through a three year course: a retrospective cohort study. Nurse Educ Pract 2017;24:14-20.

7. von Elm E, Altman DG, Egger M, et al. The strengthening the reporting of observational studies in epidemiology (STROBE) statement. Epidemiology 2007;18:800-4.

8. Rochon PA, Gurwitz JH, Sykora K, et al. Reader's guide to critical appraisal of cohort studies: 1 . Role and design. BMJ 2005;330:895-7.

9. Roever L. Critical appraisal of cohort studies. Evidence based Medicine and Practice 2015;1:e108.

10. Critical Appraisal Skills Programme. CASP (cohort study) checklist, 2018. Available: https://casp-uk.net/wp-content/ uploads/2018/03/CASP-Cohort-Study-Checklist-2018_fillable_ form.pdf [Accessed 31 July 2019]. 